Ormond Street Hospital for Children, 30 Guilford Street, London, WC1N 1EH, UK. E-mail: s.sonnappa@ich.ucl.ac.uk

Support Statement: This study was supported by Asthma UK, Smiths Medical and the European Respiratory Society.

Statement of Interest: A statement of interest for the study itself can be found at www.erj.ersjournals.com/site/misc/state ments.xhtml

\section{REFERENCES}

1 Pierse N, Rushton L, Harris RS, et al. Locally generated particulate pollution and respiratory symptoms in young children. Thorax 2006; 61: 216-220.
2 Andersen ZJ, Loft S, Ketzel M, et al. Ambient air pollution triggers wheezing symptoms in infants. Thorax 2008; 63: 710-716.

3 Nordling E, Berglind N, Melen E, et al. Traffic-related air pollution and childhood respiratory symptoms, function and allergies. Epidemiology 2008; 19: 401-408.

4 Gauderman WJ, Vora H, McConnell R, et al. Effect of exposure to traffic on lung development from 10 to 18 years of age: a cohort study. Lancet 2007; 369: 571-577.

5 Sonnappa S, Bastardo CM, Wade A, et al. Symptom-pattern phenotype and pulmonary function in preschool wheezers. J Allergy Clin Immunol 2010; 126: 519-526.

6 Gowdy K, Krantz QT, Daniels M, et al. Modulation of pulmonary inflammatory responses and antimicrobial defenses in mice exposed to diesel exhaust. Toxicol Appl Pharmacol 2008; 229: 310-319.

DOI: 10.1183/09031936.00181910

\title{
Severe hypersensitivity reaction as acute eosinophilic pneumonia and skin eruption induced by proguanil
}

\section{To the Editors:}

The combination of atovaquone and proguanil is effective and safe in the prevention and treatment of malaria. We present the first report of proguanil-induced acute eosinophilic pneumonia (AEP) associated with skin eruption. On December 18, 2006, a 40-yr-old female was admitted to our hospital (Victor Provo Medical Centre, Roubaix, France) for shortness of breath and widespread skin lesions. She had a 10-pack-yr smoking history but no respiratory illness or relevant comorbidity. On the morning of December 8, 2006, she had initiated atovaquone-proguanil treatment for malaria prophylaxis prior to a trip to Senegal. She had taken the same treatment for the first time 1 yr previously without any complications. That evening she developed progressive dyspnoea with nonproductive cough and subacute fever $\left(38^{\circ} \mathrm{C}\right)$. She only stayed in Senegal for 4 days and did not leave her hotel room during this time. After returning to France she continued to take atovaquoneproguanil for 1 week. Increased shortness of breath prompted her to consult her general practitioner, who prescribed amoxicillin plus clavulanic acid for bronchitis. This treatment was withdrawn on the day 4 because of rapidly spreading skin eruptions on day 3. Although amoxicillin plus clavulanic acid was then replaced by telithromycin, the respiratory and skin symptoms worsened and the patient was referred to our hospital.

Physical examination revealed a widespread non-pruritic maculopapular eruption, without Nikolsky sign or mucosal involvement (fig. 1a). The patient was tachypnoeic with diffuse inspiratory crackles. The superficial lymph nodes were not enlarged. A chest radiograph showed subtle, diffuse, reticulonodular opacities. High-resolution computed tomography revealed a bilateral ground-glass pattern with non-specific consolidations and linear opacities (fig. 1b). Arterial blood gas analysis (with room air) revealed oxygen tension of $68 \mathrm{mmHg}$, carbon dioxide tension of $32 \mathrm{mmHg}$ and oxygen saturation of $94 \%$. The white blood cell count on admission was $26 \times$ $10^{3}$ cells $\cdot \mathrm{mm}^{-3}$ with $11 \%$ eosinophils $\left(2.9 \times 10^{3}\right.$ cells $\left.\cdot \mathrm{mm}^{-3}\right)$. The C-reactive protein level was $136 \mathrm{mg} \cdot \mathrm{L}^{-1}$. Total serum immunoglobulin (Ig)E was $37,210 \mathrm{kU} \cdot \mathrm{L}^{-1}$. Tests for various pulmonary pathogens (Strongoloides stercoralis, Toxocara canis, Ascaris lumbricoides and Tricheinella spiralis), a urine analysis and stool tests for ova and parasites were negative. Antinuclear antibody, rheumatoid factor and HIV blood tests were all negative. On day 4 the leukocyte count peaked at $28 \times 10^{3}$ cells $\cdot \mathrm{mm}^{-3}$ ( $39 \%$ eosinophils). The patient underwent fibreoptic bronchoscopy on day 4. Bronchoalveolar lavage (BAL) showed an abundance of eosinophils: total cell count 780,000 cells $\cdot \mathrm{mL}^{-1}$; macrophages $67 \%$; lymphocytes $2 \%$; neutrophils $3 \%$; and eosinophils $28 \%$. Blood and BAL cultures were negative. A skin biopsy revealed minor cell infiltration, composed mostly of lymphocytes with some eosinophils, without vasculitis. The direct immunofluorescence assays were negative.

A drug-induced AEP was suspected and atovaquone-proguanil was withdrawn soon after hospitalisation. The patient recovered within 1 week with no need for prednisolone therapy. 2 months later, prick, intradermal and patch tests (with 30\% drug diluted in a petrolatum base) were performed according to a previously published method [1]. The patch was removed after $48 \mathrm{~h}$ and the tests were scored at 48 and $72 \mathrm{~h}$ and 8 days, according to the International Contact Dermatitis Group system [1]. Drug provocation tests and immediate and late prick, intradermal and patch skin tests for amoxicillin, ceftriaxione, erythromycin and telithromycin were negative. The patient had a doubtful skin-prick test result for atovaquone-proguanil. The patch test results for antimalarial drugs (all negative in controls) are presented in table 1. The 48- and 72-h late readings showed a strong positive reaction for atovaquone-proguanil and proguanil alone, but not atovaquone (fig. 1c). 

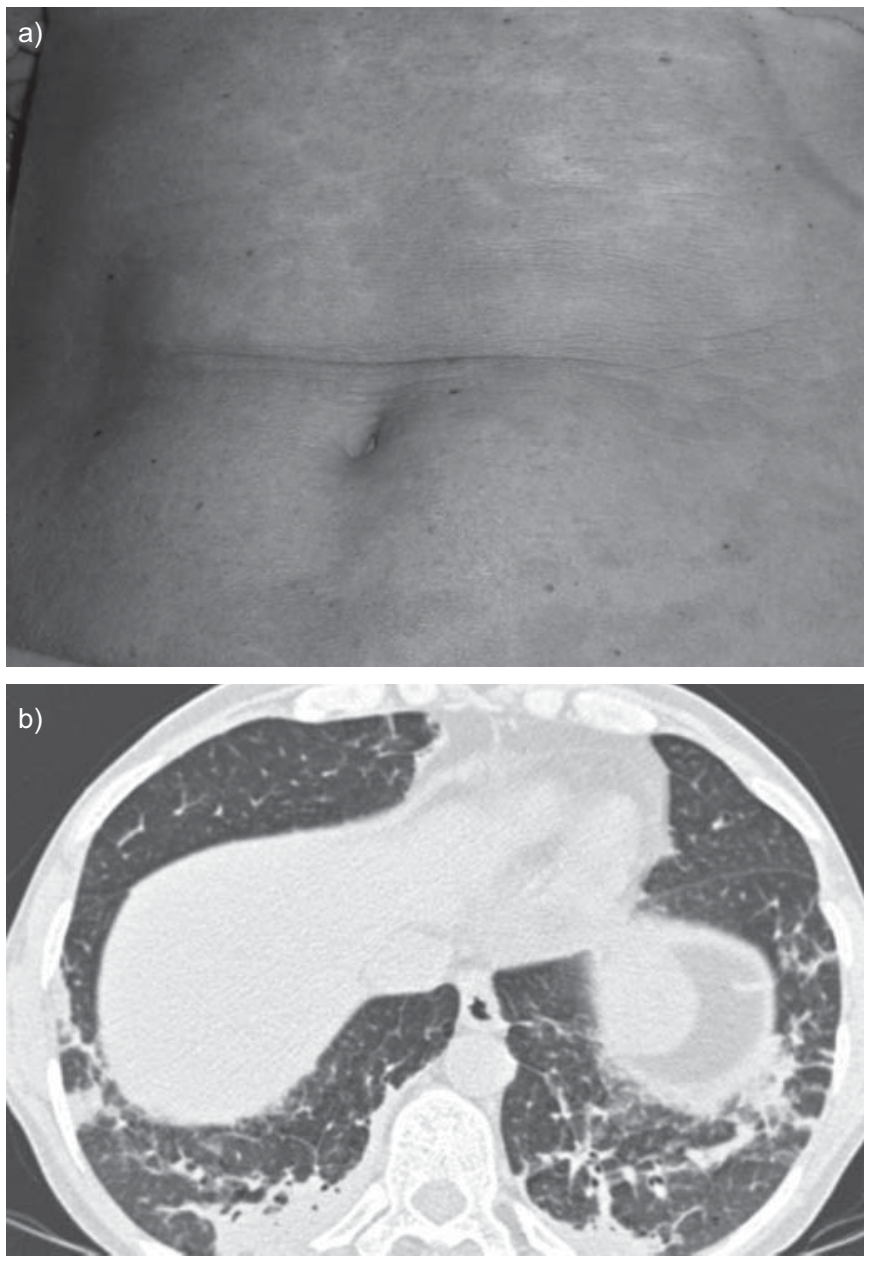

There are a few literature reports of allergy to antimalarial prophylactic drugs. The combination of chloroquine and proguanil had been suspected in a case of acute generalised exanthematous pustulosis [2] and a case of cutaneous vasculitis [3], but this has not been confirmed by tests. In this study, we have described an AEP associated with skin eruption appearing during administration of atovaquoneproguanil and confirmed the specific responsibility to proguanil by skin tests. The hypersensitivity syndrome AEP is life

\begin{tabular}{|c|c|c|c|}
\hline \multirow[t]{2}{*}{ TABLE 1} & \multicolumn{3}{|c|}{$\begin{array}{l}\text { Summary of patch test results with several } \\
\text { antimalarial drugs }\end{array}$} \\
\hline & $48 \mathrm{~h}$ & $72 \mathrm{~h}$ & 8 days \\
\hline Malarone & ++ & ++ & 0 \\
\hline Proguanil & ++ & ++ & 0 \\
\hline Atovaquone & 0 & 0 & 0 \\
\hline Quinine & 0 & 0 & 0 \\
\hline Chloroquine & 0 & 0 & 0 \\
\hline Mefloquine & 0 & 0 & 0 \\
\hline
\end{tabular}

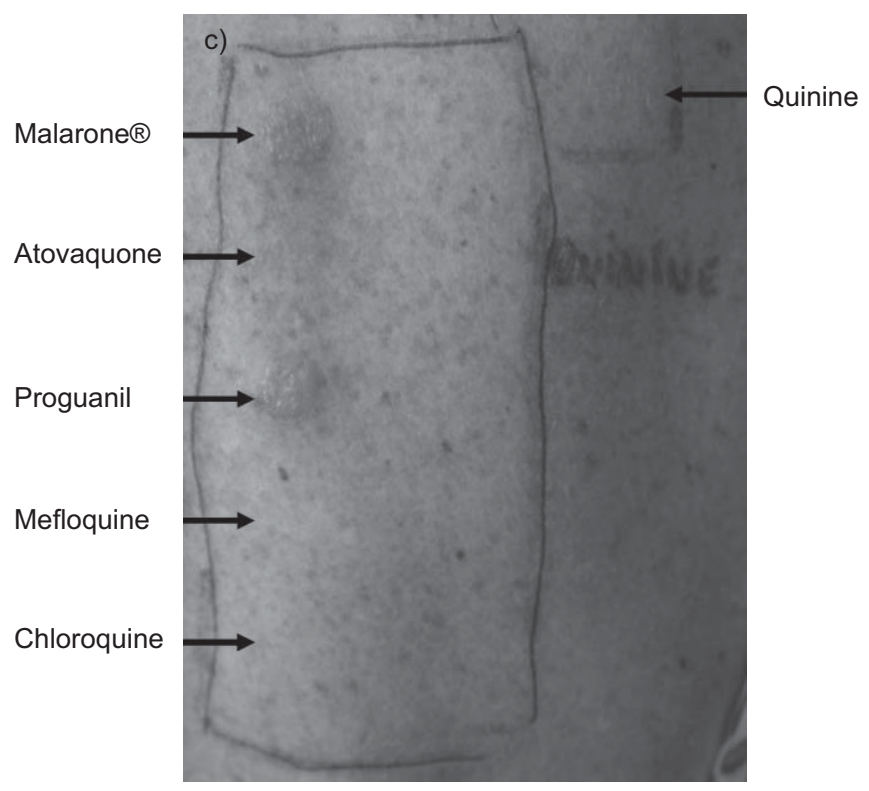

FIGURE 1. a) Widespread maculopapular rash. b) Chest computed tomography showing bilateral micronodular lesions with ground-glass and non-specific consolidations and linear opacities (mainly in the lower lobes). c) Results of drugpatch testing at day 2 for Malarone (GlaxoSmithLkine, Research Triangle Park, NC, USA), atovaquone, proguanil, mefloquine, chloroquine and quinine.

threatening and results from eosinophil infiltration of lung tissue. AEP is usually self-limiting and may be primary or caused by several factors, including drugs or parasite migration. In the present case, the parasite infection tests were negative and the patient had skin eruption, a feature which has not been seen in published AEP cases. As a result, the diagnosis of DRESS (drug rash, eosinophilia and systemic symptoms) syndrome is a possibility. DRESS is defined by fever, skin eruption, enlarged lymph nodes, visceral involvement, haematological abnormalities (hypereosinophilia and lymphocytosis) [4] and viral reactivation, in particular human herpes virus-6 and Epstein-Barr virus. DRESS syndrome has a clinical variable presentation [5] and was considered possible in the present case, according to the new RegiSCAr group's criteria [6], but not definite. Viral status had not been evaluated. IgE serum levels were particularly high but nonspecific and rapidly normalised after drug disruption. Skin tests are considered to be reliable for exploring delayed hypersensitivity reactions induced by drugs, particularly patch tests [7]. We performed skin tests to confirm the specific reaction to proguanil, as well as the absence of cross-reactivity to other drugs that may be used for prophylaxis or treatment of malaria.

In view of the widespread prophylactic use of proguanil, this observation of acute severe hypersensitivity reaction should be borne in mind by practitioners. 
N. Just*, O. Carpentier", C. Brzezinki ${ }^{\mp}$, F. Steenhouwer ${ }^{+}$and D. Staumont-Sallé

Depts of *Pneumology and Allergology, "Internal Medicine, ${ }^{+}$Pneumology, Victor Provo Medical Centre, Roubaix, and "Dept of Dermatology, Claude-Huriez Hospital, Lille University Medical Hospital, Lille, France.

Correspondence: N. Just, Service de Pneumologie, Hôpital Victor Provo, 11-17 Bd Lacordaire, F-59100 Roubaix Cedex, France. E-mail: nicolas.just@ch-roubaix.fr

Statement of Interest: None declared.

\section{REFERENCES}

1 Brockow K, Romano A, Blanca M, et al. General considerations for skin test procedures in the diagnosis of drug hypersensitivity. Allergy 2002; 57: 45-51.
2 Janier M, Froidevaux D, Lons-Danic D, et al. Acute generalized exanthematous pustulosis due to the combination of chloroquine and proguanil. Dermatology 1998; 196: 271.

3 Luong MS, Bessis D, Raison-Peyron N, et al. Severe mucocutaneous necrotizing vasculitis associated with the combination of chloroquine and proguanil. Acta Derm Venereol 2003; 83: 141.

4 Bocquet $\mathrm{H}$, Bagot M, Roujeau JC. Drug-induced pseudolymphoma and drug hypersensitivity syndrome (Drug Rash with Eosinophilia and Systemic Symptoms: DRESS). Semin Cutan Med Surg 1996; 15: 250-257.

5 Chen YC, Chiu HC, Chu CY. Drug reaction with eosinophilia and systemic symptoms: a retrospective study of 60 cases. Arch Dermatol 2010; 146: 1373-1379.

6 Kardaun SH, Sidoroff A, Valeyrie-Allanore L, et al. Variability in the clinical pattern of cutaneous side-effects of drugs with systemic symptoms: does a DRESS syndrome really exist? Br J Dermatol 2007; 156: 609-611.

7 Santiago F, Goncalo M, Vieira R, et al. Epicutaneous patch testing in drug hypersensitivity syndrome (DRESS). Contact Dermatitis 2010; 62: 47-53.

DOI: $10.1183 / 09031936.00123710$

\section{A specific point-of-care screen for infectious pleural effusions using reagent strips}

\section{To the Editors:}

The timely diagnosis of infectious pleural effusions may be challenging [1]. Infection should be suspected in patients with radiographic pleural opacities and fever. However, fever is often present not only in parapneumonics, but also in tuberculous and, less commonly, other exudative effusions. Pleural fluid analysis is the appropriate tool to aid in the differential diagnosis, but it may not be available on an emergency basis.

The dipstick leukocyte esterase test is intended to detect leukocytes in urine, but it has also been applied to other biological specimens for the rapid diagnosis of infection. The test uses the ability of the esterase enzyme present in the polymorphonuclear leukocytes of the sample to split heterocyclic carboxylates and form a pyrrole. The latter reacts with a diazonium salt producing a violet colour in the reagent strip. We hypothesised that testing pleural fluid with leukocyte esterase reagent strips may help to rule in or out a bacterial aetiology (mostly parapneumonic effusions) in just a few minutes. If this were the case, the test would be of interest to emergency departments, war-time military medical units and countries with limited medical facilities [2].

We prospectively evaluated all consecutive patients who underwent a diagnostic thoracentesis at the Arnau de Vilanova University Hospital (Lleida, Spain) from September 2009 to November 2010. The ethics committee approved the study and all patients gave written informed consent.

After the pleural tap, the fluid was sent for routine biochemical, microbiological and cytological analyses and immediately tested with Combi-Screen $₫$ (Analyticon Biotechnologies AG, Lichtenfels, Germany) reagent strips designed for urine, by an investigator blinded to the clinician diagnosis. Similarly, the clinicians were unaware of the dipstick results until the end of the study. A drop of non-centrifuged pleural fluid collected in heparinised tubes was applied to the leukocyte label of the reagent strip. Then the colour change was visually read against the colour scale on the container at precisely $2 \mathrm{~min}$. The results were recorded as: 0 (no change), $1+, 2+$ or $3+$. The finding of $1+$ or more was considered a positive test. The price of each strip was $€ 0.32$.

The aetiology of pleural effusions was established by standard clinical criteria [1]. Patients were divided into three groups, namely, nonmycobacterial infectious, tuberculous and noninfectious effusions. Complicated parapneumonic effusions refer to those non-purulent effusions associated with bacterial pneumonia which require a tube thoracostomy for cure. Empyema was defined as pus in the pleural space. Between-group comparisons of qualitative and quantitative variables were made by the Fischer exact test and Kruskal-Wallis test, respectively. The discriminative properties of reagent strips were evaluated using receiver operating characteristic curve analysis. We calculated measures of test efficacy, such as sensitivity, specificity and likelihood ratios (LR). If the $2 \times 2$ contingency contained any zeros, resulting in likelihood estimates of zero or infinity, 0.5 was added to all the counts for calculating the LR and the respective confidence intervals. The correlation between semi-quantitative dipstick results and the number of leukocytes and neutrophils in the pleural fluid was performed using the Spearman correlation coefficient. In a small subgroup of patients, weighted $\kappa$ was used to assess the level of agreement on dipstick readings.

Out of 145 patients recruited during the study period, 17 were excluded due to the presence of extremely bloody fluids, which make test strips uninterpretable, or because of an uncertain cause of the pleural effusion. The aetiological distribution of the final 128 pleural effusion patients, of whom 